\title{
DYADIC METHODS IN THE MEASURE THEORY OF NUMBERS
}

\section{BY}

\section{R. C. BAKER}

ABSTRACT. Some new theorems in metric diophantine approximation are obtained by dyadic methods. We show for example that if $m_{1}, m_{2}, \ldots$, are distinct integers with $m_{n}=O\left(n^{p}\right)$ then $\Sigma_{n<N} e\left(m_{n} x\right)=O\left(N^{1-q}\right)$ except for a set of $x$ of Hausdorff dimension at most $(p+4 q-1) /(p+2 q)$; and that for any sequence of intervals $I_{1}, I_{2}, \ldots$ in $[0,1)$ the number of solutions of $\left\{x^{n}\right\} \in I_{n}(n<N)$ is a.e. asymptotic to $\Sigma_{n<N}\left|I_{n}\right|(x>1)$.

1. Introduction. Let $g_{1}(x), g_{2}(x), \ldots$ be a sequence of differentiable functions on the finite interval $[\alpha, \beta]$. Throughout the paper we assume that $g_{1}^{\prime}(x)$ and $g_{k}^{\prime}(x)-g_{j}^{\prime}(x)$ are positive and monotonic increasing in $[\alpha, \beta]$ whenever $k>j \geqslant 1$. We also assume that there are numbers $C>0, c>0$ and $a, 0 \leqslant a<$ 1 , such that

$$
g_{k}^{\prime}(x)-g_{j}^{\prime}(x) \geqslant c
$$

whenever $j \geqslant 1$ and $k \geqslant j+C j^{a}$.

Let $f$ be an integrable function on $[0,1)$. Extend $f$ to the real line by periodicity. We write

$$
S(f, m, n, x)=\frac{1}{n} \sum_{k=m+1}^{m+n} f\left(g_{k}(x)\right)-\int_{0}^{1} f(y) d y
$$

for $m \geqslant 0, n \geqslant 1$. Let $F$ be the family of indicator functions of intervals in $[0,1)$. We write

$$
D(m, n, x)=\sup _{f \in F}|S(f, m, n, x)| .
$$

$D(m, n, x)$ is the discrepancy of $g_{m+1}(x), \ldots, g_{m+n}(x)$ (modulo 1).

THEOREM 1. Suppose that for some $p \geqslant 1-a$,

$$
g_{k}^{\prime}(\beta) \ll k^{p} \text {. }
$$

Then if $0<q<1 / 2(1-a)$, we have

$$
D(0, n, x)=O\left(n^{-q}\right)
$$

Received by the editors March 13, 1975.

AMS (MOS) subject classifications (1970). Primary 10K05, $10 \mathrm{K15}$.

Key words and phrases. Dyadic representation of integers, discrepancy modulo one, Hausdorff dimension, strong uniform distribution, fractional parts of sequences. 
except for a set of $x$ in $[\alpha, \beta]$ of Hausdorff dimension at most

$$
(p+4 q-1 / 2(1-a)) /(p+2 q+1 / 2(1-a)) .
$$

Constants implied by $\ll$ will be independent of all variables $j, k, x, \ldots$ appearing in the inequality. In [1] I obtained instead of (5) the bound

$$
(p+5 q-(1-a)) /(p+2 q) \quad(0<q<(1-a) / 3)
$$

which is sharper for small $q$. J. W. S. Cassels [4] and P. Erdös and J. F. Koksma [7] showed that (4) holds almost everywhere in $[\alpha, \beta]$ (in the Lebesgue sense) for $0<q<1 / 2(1-a)$.

Throughout the paper $\rho(n), \eta_{1}(n), \eta_{2}(n), \ldots$ are positive functions of $n=1,2, \ldots$ such that

$$
\sum_{\eta_{i}(n) / n<\infty,} \quad \sum_{\rho(n) / n}<\infty,
$$

$n \rho(n)$ is nondecreasing for large $n$, and $\eta_{t}(n)$ is nonincreasing for $n=1,2, \ldots$

THEOREM 2. Let $f$ be a square-integrable function on $[0,1)$ such that

$$
f(x) \sim \sum_{-\infty}^{\infty} c_{k} e^{2 \pi i k x}, \quad r_{m}=\sum_{k=m+1}^{\infty}\left|c_{k}\right|^{2} \ll \eta_{1}(m)
$$

for $m=1,2, \ldots$ Then

$$
S(f, 0, n, x)=o(1)
$$

for almost all $x$ in $[a, \beta]$.

The case $a=0$ of Theorem 2 is due to Koksma [10]. Koksma's theorem does not apply to $g_{j}(x)=x j^{1-a}(0<a<1)$, for example.

We write $|J|$ for the Lebesgue measure of a real set $J$.

THEOREM 3. Suppose that for $n=1,2, \ldots$

$$
\sum_{k=1}^{n} g_{k}^{\prime}(x)<g_{n}^{\prime}(x)
$$

for $x \in[\alpha, \beta]$. Let $J_{k}$ be a set in $[0,1)$, the union of $t_{k}$ disjoint intervals, for $k=1,2, \ldots$ Let $\Psi(n)=\sum_{k=1}^{n}\left|J_{k}\right|$. Let $N(n, x)$ denote the number of integers $k, 1 \leqslant k \leqslant n$, for which the fractional part $\left\{g_{k}(x)\right\}$ falls in $J_{k}$.

(a) If $t_{k}=1$ for $k=1,2, \ldots$, then for every $\epsilon>0$,

$$
N(n, x)=\Psi(n)+O\left(\Psi(n)^{1 / 2} \log ^{(3+\epsilon) / 2} \Psi(n)\right)
$$

for almost all $x$ in $[\alpha, \beta]$.

(b) If $t_{k} \ll \Psi(k) \rho([\Psi(k)])$, then 


$$
N(n, x)=\Psi(n)+o(\Psi(n))
$$

for almost all $x$ in $[\alpha, \beta]$.

(c) The conclusions (a) and (b) are valid if instead of (9) we suppose that $g_{k}(x)=\lambda_{k} x$ where $0<\lambda_{1}<\lambda_{2}<\cdots$ are integers whose greatest common divisors satisfy

$$
\sum_{j=1}^{n}\left(\lambda_{j}, \lambda_{n}\right)<<\lambda_{n}
$$

It is not hard to show that some restriction on $t_{k}$ is necessary. Let $\epsilon>0$; then

$$
t_{k} \ll<\exp \left(\Psi(k)^{1+\epsilon}\right)
$$

does not imply (11) almost everywhere when $g_{k}(x)=2^{k} x$; see $\S 5$.

Many results are known for the case $t_{k}=1(k=1,2, \ldots)$. Cassels [3] showed that in case (9) holds, $N(n, x)$ is bounded or unbounded with $\Psi(n)$ for almost all $x$. W. Philipp [13] proved (10) in the particular case $g_{k}(x)=\lambda_{k} x$, where $0<\lambda_{1}<\lambda_{2}<\cdots$ are real numbers satisfying $\inf _{k} \lambda_{k+1} / \lambda_{k}>1$. W. J. LeVeque [11] proved (11) in case (c) when $\left|J_{1}\right| \geqslant\left|J_{2}\right| \geqslant \cdots$. W. M. Schmidt [14] and V. Ennola [6] proved related but more difficult theorems.

\section{Proof of Theorem 1.}

LEMMA 1. Let $F$ be a positive piecewise differentiable function on $[\alpha, \beta]$. Suppose $F^{\prime}(x) \leqslant A(\alpha \leqslant x \leqslant \beta)$ and $\int_{\alpha}^{\beta} F(x) d x \leqslant B$. Let

$$
E=\{x \in[\alpha, \beta]: F(x) \geqslant d>0\} .
$$

There is a covering of $E$ with intervals $I_{1}, \ldots, I_{q}$ such that for $0<\gamma \leqslant 1$,

$$
\sum_{j=1}^{q}\left|I_{j}\right|^{\gamma}<<\left(1+A B d^{-2}\right)^{1-\gamma} B^{\gamma} d^{-\gamma} .
$$

The implied constant is absolute.

Proof. Cover $E$ with disjoint intervals $I_{1}, \ldots, I_{q},\left|I_{j}\right|=d / 2 A$ for $j<q$, $\left|I_{q}\right| \leqslant d / 2 A$, each $I_{j}$ meeting $E$. Clearly $F(x) \geqslant d / 2$ on each $I_{j}$, so

and so by Hölder's inequality

$$
\frac{d}{2}(q-1) \frac{d}{2 A} \leqslant \frac{d}{2} \sum_{j=1}^{q}\left|I_{j}\right| \leqslant B,
$$

$$
\sum_{j=1}^{q}\left|I_{j}\right|^{\gamma} \leqslant q^{1-\gamma}\left(\sum_{j=1}^{q}\left|I_{j}\right|\right)^{\gamma}<\left(1+A B d^{-2}\right)^{1-\gamma}\left(B d^{-1}\right)^{\gamma} .
$$

THEOREM 4. Let $D(m, n, x)$ be defined as in (2) where $F$ denotes some family of functions on $[0,1)$. Assume $D(m, n, x)$ is measurable for $m \geqslant 0$, $n \geqslant 1$ and 


$$
n D(m, n, x) \leqslant h(m, n, x) \text { for } m \geqslant 0, n \geqslant 1, \alpha \leqslant x \leqslant \beta,
$$

where $h(m, n, x)$ is positive and piecewise differentiable on $[\alpha, \beta]$. Assume that for some $\rho \geqslant 0, \sigma \geqslant 0, \nu \geqslant \rho+1, \mu \geqslant \sigma+1$,

$$
\int_{\alpha}^{\beta} h^{2}(m, n, x) d x<<k^{\rho} n^{\nu-\rho}
$$

and

$$
h(m, n, x) h^{\prime}(m, n, x)<<k^{\sigma} n^{\mu-\sigma} \quad(\alpha \leqslant x \leqslant \beta)
$$

for all $k \geqslant 1,0 \leqslant m \leqslant k, 1 \leqslant n \leqslant k$. Then if $0<\lambda<\min (1 / 2 \mu, 1 / 4(\nu+\mu))$, we have

$$
D(0, n, x)=O\left(n^{\lambda-1}\right)
$$

except for a set of $x$ of Housdorff dimension at most

$$
(\mu+\nu-4 \lambda) /(\mu-2 \lambda)
$$

ProOF. For $s \geqslant 1$ write

$$
H(s, x)=\sum_{t=1}^{s} \sum_{u=0}^{2^{s-t}-1} h^{2}\left(u 2^{t}, 2^{t-1}, x\right) .
$$

We have

$$
\int_{\alpha}^{\beta} H(s, x) d x \ll \sum_{t=1}^{s} 2^{s-t} 2^{s \rho} 2^{t(\nu-\rho)} \ll s 2^{\nu s}
$$

while

$$
\begin{aligned}
H^{\prime}(s, x) & \ll \sum_{t=1}^{s} \sum_{u=1}^{2^{s-t}-1}\left|h\left(u 2^{t}, 2^{t-1}, x\right) h^{\prime}\left(u 2^{t}, 2^{t-1}, x\right)\right| \\
& \ll \sum_{t=1}^{s} 2^{s-t} 2^{s \sigma} 2^{t(\mu-\sigma)} \ll s 2^{\mu s} .
\end{aligned}
$$

We may assume $\lambda>1 / 2 \nu$. Let $\gamma$ be a real number, $1-(2 \lambda-\nu) /(\mu-2 \lambda)<\gamma$ $<1$. Let

$$
E_{s}=\left\{x \in[\alpha, \beta]: H(s, x) \geqslant s^{-1} 2^{2 \lambda s}\right\}
$$

for $s=1,2, \ldots$ By Lemma 1 there is a covering of $E_{s}$ with intervals $I_{s j}$, $1 \leqslant j \leqslant q_{s}$, such that

$$
\begin{aligned}
\sum_{j}\left|I_{s j}\right|^{\gamma} & \ll\left(s^{4} 2^{s(\nu+\mu-4 \lambda)}\right)^{1-\gamma} s^{2 \gamma} 2^{s(\nu-2 \lambda) \gamma} \\
& <s^{4} 2^{s(\nu+\mu-4 \lambda-\gamma(\mu-2 \lambda))} .
\end{aligned}
$$

Let 


$$
W=\bigcap_{m=1}^{\infty} \bigcup_{s=m}^{\infty} E_{s}
$$

then because the series $\Sigma_{s} \Sigma_{j}\left|I_{s j}\right|^{\gamma}$ converges, there is a covering of $W$ by intervals $I_{1}, I_{2}, \ldots$ for which $\Sigma\left|I_{j}\right|^{\gamma}$ is arbitrarily small; so $W$ has dimension $\leqslant \gamma$, and hence $\leqslant(\nu+\mu-4 \lambda) /(\mu-2 \lambda)$.

It remains to show that if $x \notin W,(16)$ holds. For $s>s_{0}(x), x \notin E_{s}$. Suppose $n>2^{s_{0}}$, say $2^{s-1} \leqslant n<2^{s}$, then $s>s_{0}$. Choose an $f$ in $F$. Using the dyadic representation of $n$, we can write

$$
n S(f, 0, n, x)=\sum_{t} \sum_{u_{t}{ }^{t}+1}^{u_{t}{ }^{t}+2^{t-1}}\left(f\left(g_{k}(x)-\int_{0}^{1} f(y) d y\right)\right.
$$

where each $u_{t}$ is an integer, $0 \leqslant u_{t}<2^{s-t}$, and $t$ takes some of the values 1 , $\ldots$, s. By definition of $D(m, n, x)$ and (13),

$$
n D(0, n, x) \leqslant \sum_{t} h\left(u_{t} 2^{t}, 2^{t-1}, x\right)
$$

and by the Cauchy-Schwarz inequality,

$$
n^{2} D^{2}(0, n, x) \leqslant s \sum_{t} h^{2}\left(u_{t} 2^{t}, 2^{t-1}, x\right) \leqslant s H(s, x) .
$$

But $x \notin E_{s}$ and so

$$
n D(0, n, x)<2^{\lambda s} \leqslant 2^{\lambda} n^{\lambda} \quad\left(n>2^{s 0}\right)
$$

so that (16) holds, and Theorem 4 is proved.

To deduce Theorem 1 we require a smooth majorant for $n D(m, n, x)$. Write $e(x)=e^{2 \pi i x}$.

LEMMA 2. The discrepancy of $g_{m+1}(x), \ldots, g_{m+n}(x)$ satisfies

$$
n D(m, n, x) \ll \frac{n}{u+1}+\sum_{k=1}^{u} \frac{1}{k}\left|\sum_{j=m+1}^{m+n} e\left(k g_{j}(x)\right)\right|
$$

for every integer $u \geqslant 1$. The implied constant is absolute.

Proof. This is Theorem III of [8].

LEMMA 3. Let $p(x)$ be a real periodic integrable function of $x$ with period 1 and suppose

$$
\int_{0}^{1} p(x) d x=0, \quad P=\max _{t}\left|\int_{0}^{t} p(x) d x\right| .
$$

Let $g$ be a real function on $[\alpha, \beta]$ with monotonic derivative and $\min _{y}\left|g^{\prime}(y)\right|=$ $G>0$. Then

$$
\left|\int_{\alpha}^{\beta} p(g(y) d y)\right| \leqslant \frac{2 P}{G} .
$$


ProOF. This is Lemma 1 of [4].

LEMMA 4.

$$
\begin{array}{r}
\int_{\alpha}^{\beta}\left|\sum_{j=m+1}^{m+n} e\left(k g_{j}(x)\right)\right|^{2} d x<<n(m+n)^{a}\left(1+\frac{\log (n+1)}{k}\right) \\
(m \geqslant 0, n \geqslant 1, k \geqslant 1) .
\end{array}
$$

Proof. This is a slight variant of Lemma 3.1 of [1] using Lemma 3 above instead of the particular case $g(x)=\lambda x$ of Lemma 3.

Proof OF Theorem 1. Let $\theta$ be a positive number specified below. We apply Theorem 4 with

$$
\begin{array}{r}
h(m, n, x)=K\left(n^{1-\theta}+\sum_{k<n^{\theta}} \frac{1}{k}\left(\left|\sum_{j=m+1}^{m+n} \cos k g_{j}(x)\right|\right.\right. \\
\left.\left.+\left|\sum_{j=m+1}^{m+n} \sin k g_{j}(x)\right|\right)\right)
\end{array}
$$

which by Lemma 2 majorizes $n D(m, n, x)$ for a suitable $K>0$. By Lemma 4 and Minkowski's inequality,

while by (3)

$$
\begin{aligned}
\left(\int_{\alpha}^{\beta} h^{2}(m, n, x) d x\right)^{1 / 2} & \ll n^{1-\theta}+n^{1 / 2}(m+n)^{a / 2} \sum_{k<n^{\theta}}\left(\frac{1}{k}+\frac{\log ^{1 / 2}(n+1)}{k^{3 / 2}}\right) \\
& \ll n^{1-\theta}+n^{1 / 2}(m+n)^{a / 2} \log (n+1)
\end{aligned}
$$

$$
\begin{aligned}
h(m, n, x) h^{\prime}(m, n, x) & \ll n \log (n+1) \sum_{k<n^{\theta}} \sum_{j=m+1}^{m+n} g_{j}^{\prime}(\beta) \\
& \ll n^{2+\theta}(m+n)^{p} \log (n+1) .
\end{aligned}
$$

We now assume that $0<\theta \leqslant 1 / 2(1-a)$. In the notation of Theorem 4, we may take $\rho=a, \nu-\rho=2+\epsilon-2 \theta-a \geqslant 1, \sigma=p$ and $\mu-\sigma=2+\theta+\epsilon \geqslant 1$. Here $\epsilon$ is an arbitrary positive number. The condition

$$
\lambda<\min \left(\frac{\mu}{2}, \frac{\nu+\mu}{4}\right)=\min \left(\frac{2+\theta+p+\epsilon}{2}, \frac{4+p+2 \epsilon-\theta}{4}\right)
$$

is certainly satisfied if $\lambda \leqslant 1$ and $0 \leqslant \theta \leqslant(1-a) / 2 \leqslant p$. Obviously the quantity

$$
\frac{\mu+\nu-4 \lambda}{\mu-2 \lambda}=\frac{4+p+2 \epsilon-\theta-4 \lambda}{2+\theta+p+\epsilon-2 \lambda}
$$

is minimised by choosing the largest $\theta, \theta=1 / 2(1-a)$. Taking $\epsilon$ arbitrarily small, (16) holds outside a set of $x$ of dimension at most 


$$
(4+p-1 / 2(1-a)-4 \lambda) /(2+p+1 / 2(1-a)-2 \lambda) .
$$

Substituting $\lambda=1-q$ we obtain Theorem 1 .

Another application of Theorem 4 is following estimate of an exponential sum.

THEOREM 5. Under the hypothesis of Theorem 1,

$$
\sum_{k=1}^{n} e\left(g_{k}(x)\right)=O\left(n^{1-q}\right)
$$

except for a set of $x$ in $[\alpha, \beta]$ of dimension at most

$$
(p+4 q-(1-a)) /(p+2 q) .
$$

Proof. Apply Theorem 4 with $F$ consisting of $e(x)$ and $h(m, n, x)=$ $n D(m, n, x)$. By Lemma 4

$$
\int_{\alpha}^{\beta} h^{2}(m, n, x) d x<k^{a} n \log (n+1)
$$

and

$$
h(m, n, x) h^{\prime}(m, n, x) \ll k^{p} n^{2}
$$

for integers $m, n, k, 0 \leqslant m \leqslant k, 1 \leqslant n \leqslant k$. Take $\rho=a, \nu-\rho=1+\epsilon, \sigma=$ $p, \mu-\sigma=2$, and $\lambda=1-q$. The condition

$$
\lambda<\min \left(\frac{\mu}{2}, \frac{\mu+\nu}{4}\right)=\min \left(\frac{2+p}{2}, \frac{2+p+1+a+\epsilon}{4}\right)
$$

is satisfied because $p \geqslant 1-a$, and we obtain the required result by substitution in (17).

One can obtain $(p+4 q-(1-a)) /(p+q)$ instead of $(18)$ by the method of [1], but this is obviously less sharp.

3. Proof of Theorem 2. We follow [10]. It is enough to prove the theorem for real functions $f$ with mean value 0 . All functions $f, f_{n}$ in this section are real square integrable, with period one and mean value 0 .

LEMMA 5. If

$$
\int_{\alpha}^{\beta} n^{2} S^{2}(f, m, n, x) d x<(m+n)^{a} n^{2-a} \eta_{2}(n)
$$

for $m \geqslant 0, n \geqslant 1$, then

$$
S(f, 0, n, x)=o(1)
$$

for almost all $x$ in $[\alpha, \beta]$.

Proof. This is a special case of Theorem 7 of [9], which like Theorem 4 is proved by a "dyadic method". 
LEMMA 6. Suppose that

$$
\int_{0}^{1}\left(f(y)-f_{n}(y)\right)^{2} d y \ll \eta_{3}(n)
$$

and

$$
\int_{\alpha}^{\beta} n^{2} S^{2}\left(f_{n}, m, n, x\right) d x<(m+n)^{a} n^{2-a} \eta_{4}(n) .
$$

Then $S(f, 0, n, x)=o(1)$ for almost all $x$ in $[\alpha, \beta]$.

ProOF

$$
\begin{aligned}
\int_{\alpha}^{\beta} n^{2} S^{2}(f, m, n, x) d x \leqslant & 2 \int_{\alpha}^{\beta} n^{2} S^{2}\left(f_{n}, m, n, x\right) d x \\
& +2 \int_{\alpha}^{\beta} n^{2} S^{2}\left(f-f_{n}, m, n, x\right) d x
\end{aligned}
$$

$$
<(m+n)^{a} n^{2-a} \eta_{4}(n)+n \sum_{j=m+1}^{m+n} \int_{\alpha}^{\beta}\left(f-f_{n}\right)^{2}\left(g_{j}(x)\right) d x,
$$

applying (20) and the Cauchy-Schwarz inequality. Now apply Lemma 3 with $p(x)=\left(f-f_{n}\right)^{2}(x)-\int_{0}^{1}\left(f-f_{n}\right)^{2} d x=\left(f-f_{n}\right)^{2}-M_{n}$, say. Then $P \leqslant M_{n}$, and

$$
\begin{aligned}
& \int_{\alpha}^{\beta}\left(f-f_{n}\right)^{2}\left(g_{j}(x) d x \leqslant\left(\frac{2}{g_{j}^{\prime}(\alpha)}+\beta-\alpha\right) M_{n} \ll \eta_{3}(n),\right. \\
& \int_{\alpha}^{\beta} n^{2} S^{2}(f, m, n, x) d x<(m+n)^{a} n^{2-a}\left(\eta_{3}(n)+\eta_{4}(n)\right)
\end{aligned}
$$

and the lemma follows from Lemma 5.

LEMMA 7. Let $n \geqslant 1$, and let $t=t(n)$ and $v=v(n)$ be positive integers and $\gamma=\gamma(n)>0$. Then given $f$ there is an $f_{n}$ with the following properties.

$$
\int_{0}^{1}\left(f(y)-f_{n}(y)\right)^{2} d y<<r_{v}+v^{4} \gamma^{4}
$$

$$
\begin{aligned}
\int_{\alpha}^{\beta} n^{2} S^{2}(m, n, x) d x & \\
& <n(m+n)^{a}(\log (n+1) \log (t+1)+t)+n^{2} \gamma^{-2} r_{t} t^{-1}
\end{aligned}
$$

Proof. Let $f_{n}(x)=(1 / 2 \gamma) \int_{-\gamma}^{\gamma} f(x+y) d y$, then $f_{n}$ has period 1 and mean value 0 . Integrating term by term,

$$
f_{n}(x)=\sum_{k=-\infty}^{\infty} C_{k} e^{2 \pi i k x}, \quad C_{k}=\frac{\sin 2 \pi k \gamma}{2 \pi k \gamma} c_{k} \quad(k=1,2, \ldots)
$$

and $C_{0}=0, C_{-k}=\bar{C}_{k}$. By the Cauchy-Schwarz inequality,

$$
\sum_{k=t+1}^{\infty}\left|C_{k}\right| \leqslant \frac{1}{2 \pi \gamma}\left(\sum_{t+1}^{\infty}\left|c_{k}\right|^{2} \sum_{t+1}^{\infty} \frac{1}{k^{2}}\right)^{1 / 2} \ll \gamma^{-1} r_{t}^{1 / 2} t^{-1 / 2} \text {. }
$$


Note also that $\Sigma_{k=1}^{\infty}\left|C_{k}\right|^{2} \leqslant r_{0}$. Now

$$
\begin{aligned}
\int_{0}^{1}\left(f(y)-f_{n}(y)\right)^{2} d y & =2 \sum_{k=1}^{\infty}\left|c_{k}\right|^{2}\left(1-\frac{\sin 2 \pi k \gamma}{2 \pi k \gamma}\right)^{2} \\
& <r_{v}+\sum_{k=1}^{v}\left|c_{k}\right|^{2} k^{4} \gamma^{4}<<r_{v}+v^{4} \gamma^{4}
\end{aligned}
$$

and

$$
\begin{aligned}
n S\left(f_{n}, m, n, x\right) & =\sum_{k=-\infty}^{\infty} C_{k} \sum_{j=m+1}^{m+n} e\left(k g_{j}(x)\right), \\
\left|n S\left(f_{n}, m, n, x\right)\right| & \leqslant 2 \sum_{k=1}^{t}\left|C_{k}\right|\left|\sum_{j=m+1}^{m+n} e\left(k g_{j}(x)\right)\right|+2 n \sum_{k=t+1}^{\infty}\left|C_{k}\right|
\end{aligned}
$$

and by the Cauchyschwarz inequality and (24),

$$
n^{2} S^{2}\left(f_{n}, m, n, x\right) \ll \sum_{k=1}^{t}\left|C_{k}\right|^{2} \sum_{k=1}^{t}\left|\sum_{j=m+1}^{m+n} e\left(k g_{j}(x)\right)\right|^{2}+n^{2} \gamma^{-2} r_{t} t^{-1} \text {. }
$$

Integrating and using Lemma 4, we obtain the following estimate, which implies the lemma.

$$
\int_{\alpha}^{\beta} n^{2} S^{2}\left(f_{n}, m, n, x\right) d x<\sum_{k=1}^{t} n(m+n)^{a}\left(1+\frac{\log (n+1)}{k}\right)+n^{2} \gamma^{-2} r_{t} t^{-1} \text {. }
$$

Proof OF Theorem 2. By Lemmas 6 and 7, it suffices to choose for $n=1,2, \ldots$ the numbers $v=v(n), t=t(n)$ and $\gamma=\gamma(n)$ so that

$$
r_{v}+v^{4} \gamma^{4}<\eta_{3}(n),
$$

(26) $n(m+n)^{a}(\log (n+1) \log (t+1)+t)+n^{2} \gamma^{-2} r_{t} t^{-1}<n^{2-a}(m+n)^{a} \eta_{4}(n)$.

Choose $t=\left[n^{\lambda}\right]$ where $0<\lambda<1-a ; \gamma=n^{-\mu}$ where $0<\mu<\lambda / 2$, and $v=$ $\left[n^{\theta}\right]$, where $0<\theta<\mu$. Then since $\Sigma \eta_{1}\left(\left[n^{\theta}\right]\right) / n$ converges by the integral test, (25) follows; (26) is true also. This completes the proof of Theorem 2.

4. Proof of Theorem 3. This depends on a general lemma whose proof is another variant of the dyadic method. The underlying idea is due to Schmidt [14].

LEMMA 8. Let $E_{1}, E_{2}, \ldots$ be measurable sets in $[\alpha, \beta]$ with indicator functions $X_{1}, X_{2}, \ldots$; let $\psi(1), \psi(2)$, be numbers in $[0,1]$ such that $\Psi(n)=$ $\sum_{j=1}^{n} \psi(j) \rightarrow \infty$. Define $h_{j}(x)=X_{j}(x)-\psi(j)$. If

$$
\sum_{j=1}^{k}\left|\int_{\alpha}^{\beta} h_{j} h_{k} d x\right| \ll \psi(k)
$$

then for every $\epsilon>0$

$$
\sum_{j=1}^{n} h_{j}(x)=O\left(\Psi^{1 / 2}(n) \log (3+\epsilon) / 2 \Psi(n)\right)
$$


for almost all $x$ in $[\alpha, \beta]$.

Proof. This is a repetition almost verbatim of the proof of Theorem 3 of [12], starting at equation (3).

LEMMA 9. Let $J_{1}, J_{2}, \ldots$ be intervals in $[0,1)$ and let

$$
\left.E_{j}=\left\{x \in[\alpha, \beta]:\left\{g_{j}(x)\right)\right\} \in J_{j}\right\} .
$$

Define $h_{j}$ as in Lemma 8 with $\psi(j)=\left|J_{j}\right|$. Then for $j \geqslant 1, k \geqslant 1$,

$$
\left|\int_{\alpha}^{\beta} h_{j}(x) h_{k}(x) d x\right| \leqslant\left|J_{k}\right|\left(\frac{6}{g_{k}^{\prime}(\alpha)}+4 \int_{\alpha}^{\beta} \frac{g_{j}^{\prime}(x)}{g_{k}^{\prime}(x)} d x\right) \text {. }
$$

Proof. This is a slight variant of Lemma 2 of [3].

Proof OF Theorem $3(\mathrm{a})$. Let $h_{j}(x)$ be as in Lemma 9.

It suffices to show that (27) holds with $\psi(k)=\left|J_{k}\right|$.

But by Lemma 9 and (9),

$\sum_{j=1}^{k}\left|\int_{\alpha}^{\beta} h_{j}(x) h_{k}(x) d x\right| \leqslant\left|J_{k}\right|\left(\frac{6 k}{g_{k}^{\prime}(\alpha)}+4 \int_{\alpha}^{\beta} \sum_{j=1}^{n} \frac{g_{j}^{\prime}(x)}{g_{k}^{\prime}(x)} d x\right) \ll\left|J_{k}\right|$.

(It is obvious that $g_{k}^{\prime}(\alpha) \gg k$.)

LEMma 10. Let $h_{j}(x)$ be defined as in Lemma 8 for $j=1,2, \ldots$ Assume instead of (27) that

then

$$
\int_{\alpha}^{\beta}\left(\sum_{j=1}^{n} h_{j}(x)\right)^{2} d x<<\Psi^{2}(n) \rho([\Psi(n)]),
$$

$$
\sum_{j=1}^{n} h_{j}(x)=o(\Psi(n))
$$

for almost all $x$ in $[\alpha, \beta]$.

PrOOF. There is a sequence of integers $1 \leqslant m_{1}<m_{2}<\cdots$ such that $m_{r+1} / m_{r} \rightarrow 1$ as $r \rightarrow \infty$ and

$$
\sum_{r=1}^{\infty} \rho\left(m_{r}\right)<\infty .
$$

For a neat proof of this see $\left[5\right.$, p. 312]. Let $n_{1}<n_{2}<\cdots$ be integers such that $\left[\Psi\left(n_{r}\right)\right]=m_{r}$, then for every $\epsilon>0$,

$$
\left|\left\{x \in[\alpha, \beta]:\left|\sum_{j=1}^{n_{r}} h_{j}(x)\right|>\epsilon \Psi\left(n_{r}\right)\right\}\right| \ll \epsilon^{-2} \rho\left(m_{r}\right) .
$$

From this inequality we readily deduce that for almost all $x$ in $[\alpha, \beta]$,

$$
\sum_{j=1}^{n_{r}} h_{j}(x)=o\left(\Psi\left(n_{r}\right)\right) \quad \text { as } r \rightarrow \infty \text {. }
$$


In view of $\Psi\left(n_{r+1}\right) \sim \Psi\left(n_{r}\right),(30)$ implies (29) and Lemma 10 is proved.

Proof OF THEOREM 3(b). Write $J_{k}=J_{k}^{(1)} \cup \cdots \cup J_{k}^{\left(t_{k}\right)}$ where the $J_{k}^{(r)}$ are disjoint intervals in $[0,1)$. Define $E_{k}$ and $h_{k}$ as in Lemma 9, then

$$
h_{k}(x)=\sum_{r=1}^{t_{k}} h_{k}^{(r)}(x)
$$

where $h_{k}^{(r)}$ is defined like $h_{k}$ with $J_{k}^{(r)}$ replacing $J_{k}$. Thus

$$
\begin{aligned}
\int_{\alpha}^{\beta} h_{j}(x) h_{k}(x) d x & =\sum_{r=1}^{t_{j}} \sum_{s=1}^{t_{k}} \int_{\alpha}^{\beta} h_{j}^{(r)} h_{k}^{(s)} d x \\
& \leqslant t_{j} \sum_{s=1}^{t_{k}}\left|J_{k}^{(s)}\right|\left(\frac{6}{g_{k}^{\prime}(\alpha)}+4 \int_{\alpha}^{\beta} \frac{g_{j}^{\prime}(x) d x}{g_{k}^{\prime}(x)}\right)
\end{aligned}
$$

and

$$
\begin{aligned}
\sum_{k=1}^{n} \sum_{j=1}^{n}\left|\int_{\alpha}^{\beta} h_{j}(x) h_{k}(x) d x\right| & \ll \Psi(n) \rho([\Psi(n)]) \sum_{k=1}^{n}\left|J_{k}\right| \\
& \ll \Psi^{2}(n) \rho([\Psi(n)])
\end{aligned}
$$

by the hypotheses on $t_{j}$ and $\rho(n)$. Theorem 3(b) now follows on application of Lemma 10.

LEMMA 11. Let $0<\lambda_{1}<\lambda_{2}<\cdots$ be a sequence of integers. Let $J_{1}$, $J_{2}, \ldots$ be intervals in $[0,1)$. Define $h_{j}$ as in Lemma 9 with $g_{j}(x)=\lambda_{j} x$. Then for $j \geqslant 1, k \geqslant 1$,

$$
\left|\int_{0}^{1} h_{j}(x) h_{k}(x) d x\right| \leqslant \frac{2\left(\lambda_{j}, \lambda_{k}\right)}{\lambda_{k}}\left|J_{k}\right| .
$$

Proof. This is essentially the result of section 1 of [11]. For a different proof see Lemma 10 of [14] where Schmidt's argument easily adapts to prove our lemma.

Theorem 3(c) can now be proved in the same way as parts (a) and (b) by using (31) instead of (27). Lemma 8 can also be used to prove

THEOREM 6. Let $0 \leqslant \psi_{r}(j) \leqslant 1(r=1, \ldots, h, j=1,2, \ldots)$ where $h$ is a positive integer. Suppose $N\left(n, x_{1}, \ldots, x_{h}, \theta_{1}, \ldots, \theta_{h}\right)$ denotes the number of solutions of $\left\{j x_{r}-\theta_{r}\right\}<\psi_{r}(j)$ for $r=1,2, \ldots, h$ with $1 \leqslant j \leqslant n$. Let $\epsilon>0$, then for almost all $\left(x_{1}, \ldots, x_{h}, \theta_{1}, \ldots, \theta_{h}\right)$ in the sense of Lebesgue measure in $R^{2 h}$,

$$
N\left(n, x_{1}, \ldots, x_{h}, \theta_{1}, \ldots, \theta_{h}\right)=\Psi(n)+O\left(\Psi^{1 / 2}(n) \log ^{3 / 2+e} \Psi(n)\right) .
$$

Here $\Psi(n)=\Sigma_{j=1}^{n} \psi_{1}(j) \cdots \psi_{h}(j)$.

This is a quantitative version of a theorem of Cassels [2] that $N\left(n, x_{1}, \ldots, x_{h}, \theta_{1}, \ldots, \theta_{h}\right)$ is bounded or unbounded with $\Psi(n)$ almost 
everywhere. The proof is obtained by adjoining Lemma 8 to the calculations in [2], and may be left to the reader.

5. Two examples. We first give a new example of a sequence of integers $0<\lambda_{1}<\lambda_{2}<\cdots$ satisfying (12). Several examples were given in [11]; in particular (12) holds if $\lambda_{j}$ has $O(1)$ divisors.

Let $p_{1}, \ldots, p_{t}$ be a given finite set of primes and let $\left(\lambda_{j}\right)$ be the sequence of integers $p_{1}^{x_{1}} \ldots p_{t}^{x_{t}}\left(x_{1} \geqslant 0, \ldots, x_{t} \geqslant 0\right.$ integers $)$ enumerated in increasing order. We fix $\lambda_{k}=p_{1}^{a_{1}} \cdots p_{t}^{a_{t}}$, and let $\lambda_{j}=p_{1}^{x_{t}} \cdots p_{t}^{x_{t}} \leqslant \lambda_{k}$.

For reasons of symmetry we need only sum $\left(\lambda_{j}, \lambda_{k}\right) \lambda_{k}^{-1}$ over those $\lambda_{j}$ with

$$
x_{1}>a_{1}, \ldots, x_{q}>a_{q}, x_{q+1} \leqslant a_{q+1}, \ldots, x_{t} \leqslant a_{t}
$$

where $0 \leqslant q<t$. Let $M=M\left(x_{q+1}, \ldots, x_{t}\right)$ be the number of lattice points $\left(x_{1}, \ldots, x_{q}\right)$ which satisfy $(32)$ and

$$
\begin{aligned}
\left(x_{1}-a_{1}\right) & \log p_{1}+\cdots+\left(x_{q}-a_{q}\right) \log p_{q} \\
& \leqslant\left(a_{q+1}-x_{q+1}\right) \log p_{q+1}+\cdots+\left(a_{t}-x_{t}\right) \log p_{t} .
\end{aligned}
$$

We have

$$
\begin{aligned}
M & \ll\left(\left(a_{q+1}-x_{q+1}\right)+\cdots+\left(a_{t}-x_{t}\right)\right)^{q} \\
& \ll\left(a_{q+1}-x_{q+1}+1\right)^{q} \cdots\left(a_{t}-x_{t}+1\right)^{q}
\end{aligned}
$$

where the implied constant depends only on $p_{1}, \ldots, p_{t}$. But clearly, where $\lambda_{j}$ takes all values permitted by (32),

$$
\begin{aligned}
\sum_{j}\left(\lambda_{j}, \lambda_{k}\right) \lambda_{k}^{-1} & =\sum_{0<x_{r}<a_{r}(r=q+1, \ldots, t)} M\left(x_{q+1}, \ldots, x_{t}\right) p_{q+1}^{x_{q+1}-a_{q+1}} \ldots p_{t}^{x_{t}-a_{t}} \\
& \ll \prod_{r=q+1}^{t} \sum_{y=0}^{a_{r}}(y+1)^{q} p_{r}^{-y}<<1,
\end{aligned}
$$

which establishes (12).

Our other example is a complement to Theorem 3.

THEOREM 7. If $0<\lambda_{1}<\lambda_{2}<\cdots$ is a sequence of integers such that $\lambda_{j} \mid \lambda_{j+1}(j \geqslant 1)$ there is a finite union $J_{j}$ of disjoint intervals in $[0,1)$ for $j=$ $1,2, \ldots$ such that $\Psi(n)=\Sigma_{j=1}^{n}\left|J_{j}\right| \rightarrow \infty$, but the inequalities

$$
\left\{\lambda_{j} x\right\} \in J_{j}
$$

have at most finitely many solutions $j$ for almost all real $x$.

Proof. Write $n^{-1} A=\{x \in[0,1):\{n x\} \in A\}$ if $A$ is a measurable set 
in $[0,1)$ and $n$ is a positive integer. Notice that $\left|n^{-1} A\right|=|A|$.

Let $1<a<2$. Define $I_{k}=\left[0,1 / 2(k+1)^{a}\right](k=0,1, \ldots)$. If $k^{2}<$ $j \leqslant(k+1)^{2}$ for some $k \geqslant 0$ define

$$
J_{j}=\left(\lambda_{(k+1)^{2}} / \lambda_{j}\right)^{-1} I_{k} \text {. }
$$

Then $J_{j}$ is a finite union of disjoint intervals in $[0,1)$ and

$$
\sum_{j}\left|J_{j}\right|=\sum_{k} \frac{(k+1)^{2}-k^{2}}{2(k+1)^{a}}=\infty .
$$

On the other hand (33) has infinitely many solutions precisely when

$$
\left\{\lambda(k+1)^{2} x\right\} \in I_{k}
$$

has infinitely many solutions $k$, and Theorem 7 now follows from the BorelCantelli lemma.

Suppose in Theorem 7 that $\lambda_{j}=2^{j}$. Then if $k^{2}<j \leqslant(k+1)^{2}, J_{j}$ consists of $2^{(k+1)^{2}-j}<2^{2 j \frac{1}{2}}$ intervals, while

$$
\Psi(j) \gg \sum_{r<k} r^{1-a} \gg k^{2-a} \gg j^{(2-a) / 2} \text {. }
$$

Thus in the notation of Theorem 3,

$$
t_{j}<e^{\Psi(j)^{1+\delta}}
$$

where $\delta(a) \rightarrow 0$ as $a \rightarrow 1$. It would be interesting to close the gap between (34) and $t_{j} \ll \Psi(j) \rho([\Psi(j)])$.

\section{REFERENCES}

1. R. C. Baker, Slowly growing sequences and discrepancy modulo one, Acta Arith. 23 (1973), 279-293. MR 47 \# 8473.

2. J. W. S. Cassels, Some metrical theorems in diophantine approximation. I, Proc. Cambridge Philos.Soc. 46 (1950), 209-218. MR 12, 162.

3. - Some metrical theorems of Diophantine approximation. II, J. London Math. Soc. 25 (1950), 180-184. MR 12, 162.

4. - Some metrical theorems of Diophantine approximation. III, Proc. Cambridge Philos. Soc. 46 (1950), 219-225. MR 12, 162.

5. H. Davenport, P. Erdos and W. J. LeVeque, On Weyl's criterion for uniform distribution, Michigan Math. J. 10 (1963), 311-314. MR 27 \# 3618.

6. V. Ennola, On metric diophantine approximation, Ann. Univ. Turku. Ser. A I 113 (1967), 3-8. MR 37 \# 162.

7. P. Erdos and J. R. Koksma, On the uniform distribution modulo one of sequences $(f(n, \theta))$, Nederl. Akad. Wetensch. Proc. 52, 851-854 = Indag. Math. 11 (1949), 299302. MR 11, 331.

8. P. Erdøs and P. Turán, On a problem in the theory of uniform distribution. I, II, Nederl. Akad. Wetensch. Proc. 51, 1146-1154, 1262-1269 = Indag. Math. 10 (1948), 370-378, 406-413. MR 10, 372.

9. I. S. Gál and J. F. Koksma, Sur l'ordre de grandeur des fonctions sommables, Nederl. Akad. Wetensch. Proc. 53, 638-653 = Indag. Math. 12 (1950), 192-207. MR 12, 86. 
10. J. F. Koksma, An arithmetical property of some summable functions, Nederl. Akad. Wetensch. Proc. 53, 959-972 = Indag. Math. 12 (1950), 354-367. MR $12,86$.

11. W. J. LeVeque, On the frequency of small fractional parts in certain real sequences. III, J. Reine Angew. Math. 202 (1959), 215-220. MR 22 \# 12090.

12. W. Philipp, Some metrical theorems in number theory, Pacific J. Math. 20 (1967), 109-127. MR 34 \# 5755.

13. - Some metrical theorems in number theory. II, Duke Math. J. 37 (1970), 447-458. MR 42 \# 7620; errata, 43 \# 177.

14. W. M. Schmidt, Metrical theorems on fractional parts of sequences, Trans. Amer. Math. Soc. 110 (1964), 493-518. MR 28 \# 3018.

DEPARTMENT OF MATHEMATICS, ROYAL HOLLOWAY COLLEGE, EGHAM, SURREY, UNITED KINGDOM 